\title{
The Association of Introducing a Faecal \\ Calprotectin Testing Pathway for Suspected Inflammatory Bowel Disease in Primary Care and Time to Diagnosis or Treatment
}

\author{
Amy Hicks $^{a}$ P. John Hamlin ${ }^{a, b}$ Christian P. Selinger ${ }^{a, b}$

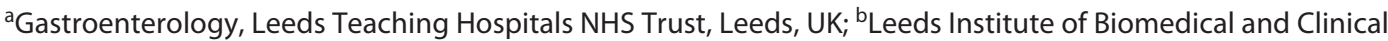 \\ Sciences, University of Leeds, Leeds, UK
}

\section{What Is Already Known?}

- Many patients with inflammatory bowel disease (IBD) experience long delays from initial symptoms to diagnosis.

- Faecal calprotectin (FC) can help distinguishing IBD from irritable bowel syndrome in primary care patients presenting with diarrhoea.

- Primary care FC testing can avoid unnecessary invasive investigations.

\section{What Is New?}

- IBD patients present via a variety of pathways and referral sources, with only a minority being referred directly to gastroenterology for evaluation of possible IBD.

- The proportion of newly diagnosed patients where calprotectin was used in the diagnostic workup prior to secondary care referral is only $37 \%$.

- The introduction of primary care FC testing was not associated with a reduction in time from referral to diagnosis.

\section{Keywords}

Ulcerative colitis · Crohn's disease · Inflammatory bowel disease

\section{Abstract \\ Background: Primary care faecal calprotectin (FC) was intro- duced in Leeds in 2014 to distinguish inflammatory bowel disease (IBD) from irritable bowel syndrome and with the hope that it may reduce time to IBD diagnosis and treatment. This study examines the association of FC with referral}

karger@karger.com www.karger.com/iid

Karger $\stackrel{\text { ' }}{5}$ BOPEN ACCESS
(C) 2020 The Author(s)

Published by S. Karger AG, Basel

This article is licensed under the Creative Commons AttributionNonCommercial-NoDerivatives 4.0 International License (CC BY NC-ND) (http://www.karger.com/Services/OpenAccessLicense) Usage and distribution for commercial purposes as well as any distribution of modified material requires written permission. routes, time to diagnosis, and time to treatment. Methods: All patients newly referred to IBD clinics in 2013 and 2016 were studied. Data on referral routes and dates, FC, date of first treatment, and proxy outcomes for disease severity were collected. Results: In 248 patients, there were no differences between 2013 and 2016 cohorts regarding baseline data and disease severity. The number of direct referrals to gastroenterology rose from 3\% (2013) to 17\% (2016), whilst $10 \%$ were diagnosed during emergency admissions. Referrals via suspected cancer pathways remained high (38\% in 2013, 28\% in 2016), whilst many had initial investigations at 
independent centres (16\% in 2013, 24\% in 2016). Time from referral to diagnosis was similar between 2013 ( 0.77 month) and 2016 (1.10 months, $p=0.2$ ). A total of 48 (33.3\%) patients had FC checked prior to referral, and $37.5 \%$ of these were referred directly to gastroenterology. Time from diagnosis to treatment reduced from 1.37 months (2013) to 0.72 month $(2016, p=0.01)$. Conclusion: Patients present via a multitude of referral pathways, but FC was associated with increased direct referrals to gastroenterology. We found a variation in time to diagnosis and treatment depending on referral routes. Further work is required to ensure patients with suspected IBD get referred to IBD services in a timely manner.

(C) 2020 The Author(s)

Published by S. Karger AG, Basel

\section{Background}

Inflammatory bowel disease (IBD), broadly subdivided into Crohn's disease (CD) and ulcerative colitis (UC), affects up to 620,000 people in the UK [1]. It is a significant chronic disease that can lead to serious complications, such as the need for major surgery and the development of colorectal cancer [2]. Prompt diagnosis of IBD is necessary to manage patients appropriately and reduce the likelihood of such complications.

Traditionally, clinical symptoms and blood tests (full blood count, C-reactive protein, and erythrocyte sedimentation rate) have been used to guide the need for referral, but these are poor at differentiating between irritable bowel syndrome (IBS) and IBD, and delays in IBD diagnosis are well documented [3-5]. In a survey of 1,121 IBD patients, $68 \%$ self-reported a delay in their diagnosis, with $48.1 \%$ reporting a delay $>2$ years [6]. Patients with $\mathrm{CD}$ have notably longer delays than those with UC $[4,6,7]$. FC - a protein released by white blood cells, indicative of inflammation within the bowel - is now recommended by NICE to help distinguish between IBD and IBS and guide referral to secondary care [8-10].

Following the publication of the NICE guidelines in 2013, FC testing in primary care was introduced in Leeds in 2014 to help distinguish IBD from IBS, with the additional aim of reducing time to IBD diagnosis and treatment by filtering out unnecessary referrals and triaging appropriate referrals straight to "test first" or urgent clinical appointment rather than a routine clinical appointment as the first contact with the patient. Several studies have examined the ability of FC testing to distinguish IBD from IBS, the effects on referral to secondary care, and the diagnostic accuracy in the setting of various gastrointestinal symptoms [11-15]. However, no study to date has examined whether primary care FC testing can reduce the time to diagnosis of IBD. This study examines the association of FC testing with time to diagnosis and treatment of IBD, as well as the association with referral routes.

\section{Methods}

The study examined data from new referrals to the IBD service at Leeds Teaching Hospitals NHS Trust. This is one of the largest trusts in the NHS with approximately 2,500 inpatient beds, serving the immediate population of 770,000 . The Leeds IBD service cares for approximately 4,000 patients with IBD.

Primary care FC testing and amended referral pathways (Fig. 1) were introduced in 2014. All patients with a new referral and diagnosis of IBD made during the whole calendar years of 2013 and 2016 (pre- and post-FC introduction, respectively) were eligible for the study and were identified from the clinic administration system. The diagnosis of IBD was based on standard endoscopic, histological, and radiology criteria. The electronic patient record $(\mathrm{PPM}+)$ for each individual was reviewed, and only those with a new diagnosis of IBD were included. Patients who were previously diagnosed with IBD and registered as new due to relocation or re-referral were excluded, as well as those patients for whom the final diagnosis was not IBD.

The initial referral letter, clinic letters, and investigation dates and results were accessed on the online patient record $(\mathrm{PPM}+)$. Data on baseline demographics (age, gender, and smoking status), symptoms and their duration, referral routes and dates, FC measurements, endoscopy reports, imaging results, IBD diagnosis subtype and extent at diagnosis, admission within the first year post-diagnosis, and date of first treatment and proxy outcomes for disease severity during the first year (steroid use, biologic use, and surgery) were collected for each patient. Date of diagnosis was classified as date of endoscopic evidence of IBD or radiological findings indicative of IBD (mostly CT imaging).

The primary study outcome was as follows:

- Time from referral to diagnosis stratified by the use of FC.

The secondary study outcomes were as follows:

- Time from diagnosis to treatment stratified by the use of FC (to assess for differences in time to treatment according to referral pathways).

- The percentage of patients undergoing FC testing.

- Sources of referral leading to IBD diagnosis.

- Proxy outcomes of disease severity (steroid use, biologic use, and surgery at 1 year) stratified by different referral sources or the use of FC.

Statistical analysis was performed using IBM SPSS Statistics 25. Baseline demographics were compared using Pearson's $\chi^{2}$ test, Fisher's exact test, independent samples $t$ test, and ANOVA, as appropriate. Time to diagnosis and treatment was compared using independent samples $t$ tests. Disease severity was analysed using the $\chi^{2}$ test. 



*Following a 3,000 patients audit the cut-offs for referral have been subsequently revised to <100: manages as IBS in primary care

101-250: repeat after 4 weeks and if 101-250 again routine gastroenterology referral

>250: urgent referral to gastroenterology for colonoscopy first

Fig. 1. Referral pathways for suspected IBD. IBD, inflammatory bowel disease.

\section{Results}

\section{Patient Characteristics}

A total of 762 patients across both cohorts were referred to the IBD service. Only 248 of these had a new diagnosis of IBD and were included in the study; 104 of these patients were referred in 2013, and there were 144 referrals in 2016 (Fig. 2).

There were no significant differences in the baseline demographics, diagnosis, and smoking status of patients in each cohort. Patients had a mean age of 43 years, with a 50:50 male-to-female ratio, and $17 \%$ were current smokers. There were no differences in age and gender when analysed by disease subtype, but the proportion of current smokers was 2- to 3 -fold higher in those diagnosed with CD (Table 1).

\section{Time to Diagnosis}

Time from referral to diagnosis of IBD was similar between 2013 (0.77 month) and 2016 (1.10 months, $p=0.2$; Table 2). Time to diagnosis was marginally longer in those who had FC tested than in those without (1.47 vs. 0.86 month, $p=0.06)$, but this was not statistically significant.

We observed notable differences in the referral sources of newly diagnosed IBD patients between 2013 and 
Table 1. Baseline demographics stratified by disease subtype (excluding indeterminate colitis) and year of diagnosis

\begin{tabular}{|c|c|c|c|c|c|c|}
\hline \multirow[t]{2}{*}{ Demographics } & \multicolumn{2}{|l|}{2013 cohort } & \multirow[t]{2}{*}{$p$ value } & \multicolumn{2}{|l|}{2016 cohort } & \multirow[t]{2}{*}{$p$ value } \\
\hline & $\mathrm{CD}(n=36)$ & $\mathrm{UC}(n=62)$ & & $\mathrm{CD}(n=49)$ & $\mathrm{UC}(n=85)$ & \\
\hline Gender, $n(\%)$ & & & 0.232 & & & 0.176 \\
\hline Male & $17(47)$ & $37(60)$ & & $20(41)$ & $45(53)$ & \\
\hline Female & $19(53)$ & $25(40)$ & & $29(59)$ & $40(47)$ & \\
\hline Mean age $\pm S D$, years & $41.6 \pm 16.4$ & $45.0 \pm 16.0$ & 0.324 & $42.9 \pm 19.6$ & $42.1 \pm 17.2$ & 0.798 \\
\hline Smoking status, $n(\%)$ & & & 0.026 & & & 0.017 \\
\hline Yes & $12(33)$ & $6(10)$ & (for yes vs. other & $10(20)$ & $8(9)$ & \\
\hline No & $13(36)$ & $25(40)$ & groups combined) & $20(41)$ & $26(31)$ & \\
\hline Ex-smoker & $6(17)$ & $15(24)$ & & $10(20)$ & $19(22)$ & \\
\hline Unknown & $5(14)$ & $16(26)$ & & $9(18)$ & $32(38)$ & \\
\hline
\end{tabular}

CD, Crohn's disease; UC, ulcerative colitis. Bold values indicate significance.

Fig. 2. Patient flow in the study cohort. IBD, inflammatory bowel disease.

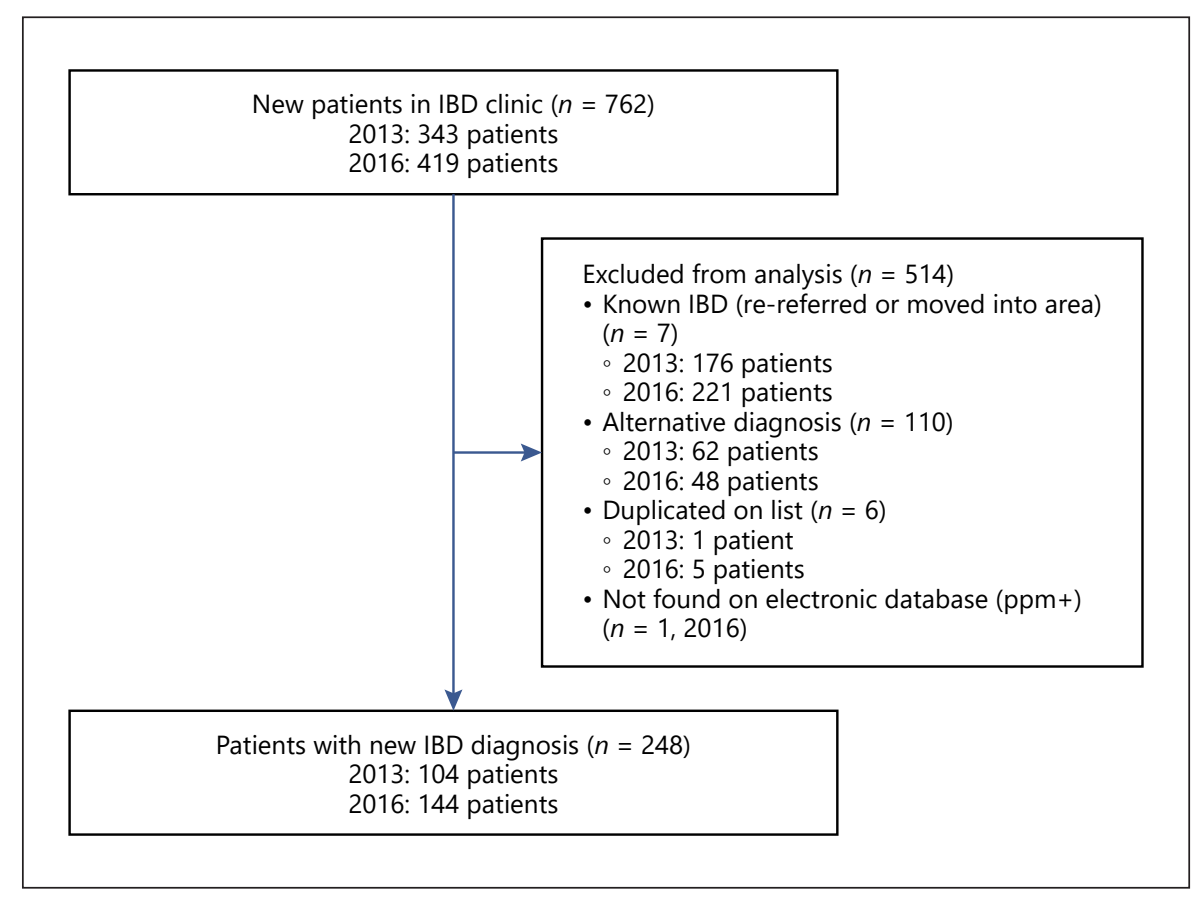

2016. The routes of initial referral to secondary care and their association with time to diagnosis and treatment are displayed in Table 3. The number of patients referred directly to gastroenterology rose from $3 \%$ (2013) to $17 \%$ (2016); 10\% of diagnoses were made during emergency admissions to hospital (unchanged). Referrals via the 2 -week wait suspected cancer pathway remained high (38\% in 2013, 28\% in 2016), whilst many had initial investigations at independent centres contracted to provide NHS care (16\% in $2013,24 \%$ in 2016$)$.
Although overall time from referral to diagnosis of IBD increased non-significantly from 2013 to 2016, this result appears to be influenced by individual referral routes. Data on time to diagnosis of IBD were unfortunately unavailable for those who underwent endoscopic procedures at independent NHS providers. Time to diagnosis was similar across all referral routes except for those referred to surgical specialties (2.0 vs. 3.8 months, $p=$ 0.220 ) and those referred via private care (note only 1 referral via this route in 2013). 
FC was checked in 48 patients prior to referral, and $37.5 \%$ of these were referred directly to gastroenterology. There were no significant differences in time to diagnosis between those patients with a FC and those without (see online suppl. Table 1; for all online suppl. material, see www.karger.com/doi/10.1159/000509907).

In all, $73 \%$ of patients had a $\mathrm{FC}>600 \mu \mathrm{g} / \mathrm{g}$, yet only $37.1 \%$ of these were referred directly to gastroenterology (23.7\% were referred to private services, $13.2 \%$ were hospital admissions, and $26 \%$ were referred to other services). Of the patients referred directly to gastroenterology who had FC checked (Table 3), $72 \%$ had a level $>600$. It is noted that of the 48 patients with FC measured, 12 did not have results available at the time of referral (also note 17 patients had an unknown referral date). Of those $19 \mathrm{pa}-$ tients who had results available at the time of referral, $63 \%$ were referred directly to gastroenterology.

\section{Time to Treatment}

Time from diagnosis to treatment reduced from 1.37 months $(2013)$ to 0.72 month $(2016, p=0.01)$. Time from diagnosis to treatment when FC was checked was also numerically shorter $(0.78$ month with FC vs. 1.04 months without, $p=0.383$ ). No treatment was started at the initial clinic visit for 14 patients (conservative/expectant approach); these were excluded from the time to treatment analyses.

Time from diagnosis to treatment has improved across all referral routes except for those patients referred to surgical specialties ( 1.0 vs. 1.62 months, $p=0.513$; online suppl. Table 1) and those referred via private care (only 1 referral via private care in 2013). The only referral route with a statistically significant difference in time to treatment is the bowel cancer screening group (5.0 vs. 1.67 months, $p=0.034)$. The shortest times to treatment occurred in patients seen directly by gastroenterologists (0.67 month in $2013,0.44$ month in 2016 ) or when admitted to hospital (0.8 month in 2013, 0.31 month in 2016).

\section{The Association of Age with Pathways and Time to}

\section{Diagnosis and Treatment}

Patients over the age of 40 years were referred via 2 -week wait cancer pathways more frequently than patients under 40 years (60\% of referrals over 40 years vs. $3 \%$ of referrals under 40 years, $p<0.001$ ). Time to diagnosis was significantly shorter in patients aged $\geq 66$ years (2016: mean of 0.4 month) than those aged $\leq 40$ years (2016: mean of 1.5 months, $p=0.001$; online suppl. Tables $2,3)$. Time to treatment however was significantly longer in patients aged $\geq 66$ years (2016: mean of 1.4 months)

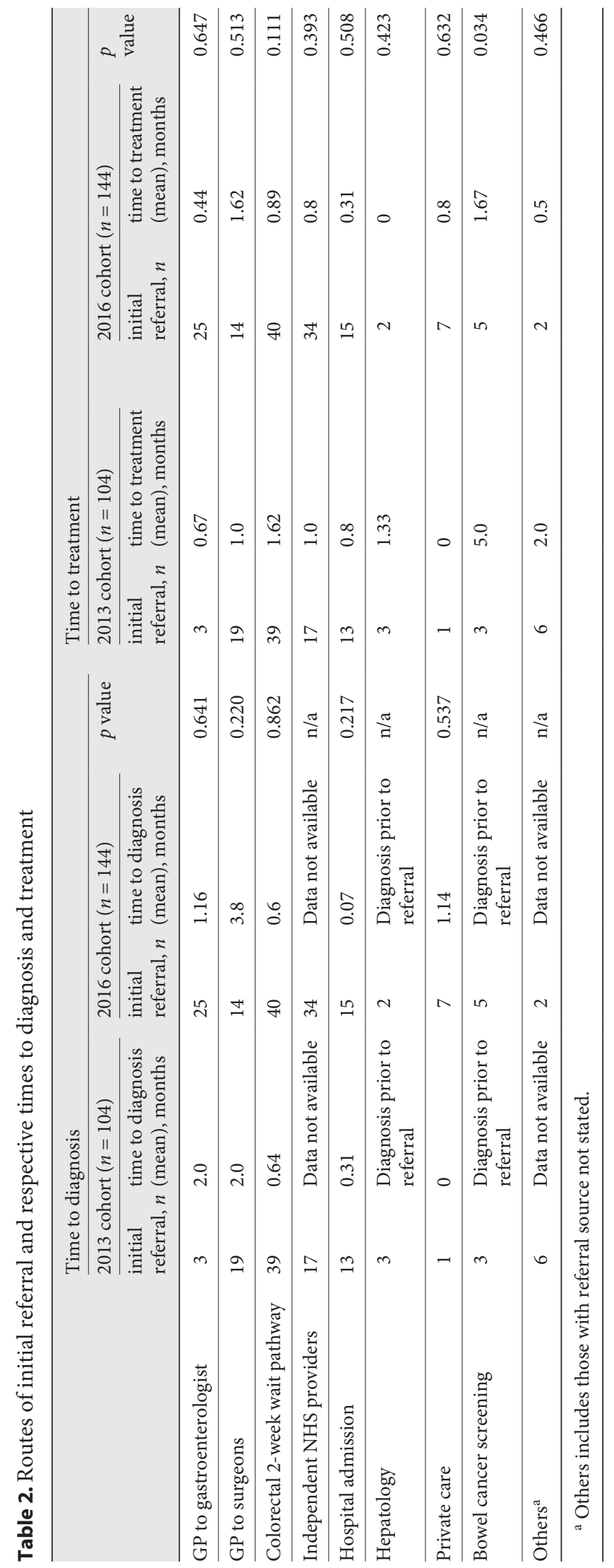

Inflamm Intest Dis 2020;5:191-199 DOI: $10.1159 / 000509907$ 
Table 3. Number with FC: 48 (+2 requested by GP)

\begin{tabular}{|c|c|c|c|c|}
\hline FC level & $\begin{array}{l}\text { Cases, } \\
n\end{array}$ & $\begin{array}{l}\text { FC level to diagnosis } \\
\text { (mean months } \\
\text { [number included } \\
\text { in analysis]) }\end{array}$ & $\begin{array}{l}\text { Referral to diagnosis } \\
\text { (mean months } \\
\text { [number included } \\
\text { in analysis]) }\end{array}$ & $\begin{array}{l}\text { Route of referral } \\
\text { (mean months referral } \\
\text { to diagnosis [number } \\
\text { included in analysis]) }\end{array}$ \\
\hline$>600 \mu \mathrm{g} / \mathrm{g}$ & 35 & $\begin{array}{l}2.56[32] \\
\text { NB. } 2.0 \\
\text { (if exclude one outlier) }\end{array}$ & $1.41[22]$ & $\begin{array}{l}\text { GP to Gastro }-1.39[13] \\
\text { 2WW - } 1.0[2] \\
\text { Admission }-0.33[3] \\
\text { Independent }-\mathrm{n} / \mathrm{a}[11] \\
\text { Private }-2.5[2] \\
\text { GP to surgeons }-\mathrm{n} / \mathrm{a}[1] \\
\text { Not stated }-\mathrm{n} / \mathrm{a}[1]\end{array}$ \\
\hline $250-600 \mu \mathrm{g} / \mathrm{g}$ & 6 & 2.17 & $1.0[4]$ & $\begin{array}{l}\text { GP to Gastro }-1.0[2] \\
\text { Independent }-\mathrm{n} / \mathrm{a}[2] \\
\text { Private }-1.0[1] \\
2 \mathrm{WW}-1.0[1]\end{array}$ \\
\hline $100-250 \mu \mathrm{g} / \mathrm{g}$ & 3 & 2.5 & $0.5[2]$ & $\begin{array}{l}\text { Gastro }-0.5[2] \\
\text { Independent }-\mathrm{n} / \mathrm{a}[1]\end{array}$ \\
\hline$<100 \mu \mathrm{g} / \mathrm{g}$ & 3 & 9.67 & $6.0[1]$ & $\begin{array}{l}\text { Independent }-\mathrm{n} / \mathrm{a}[2] \\
\mathrm{GP} \text { to surgeons }-6.0[1]\end{array}$ \\
\hline "Elevated" & 1 & $\mathrm{n} / \mathrm{a}$ & 1.0 & GP to Gastro - $1.0[1]$ \\
\hline
\end{tabular}

FC, faecal calprotectin.

Table 4. Proxy outcomes of disease severity

\begin{tabular}{lccl}
\hline & $\begin{array}{l}2013 \text { cohort } \\
(n=104)\end{array}$ & $\begin{array}{l}2016 \text { cohort } \\
(n=144)\end{array}$ & $\begin{array}{l}\chi^{2} \\
(p \text { value })\end{array}$ \\
\hline Steroids at diagnosis, \% & 33.7 & 39.6 & 0.340 \\
Surgery in the first year, \% & 6.7 & 5.6 & 0.702 \\
Biologics in the first year, \% & 8.3 & 16.1 & 0.128 \\
\hline
\end{tabular}

than those aged $\leq 40$ years (2016: mean of 0.6 month, $p=$ 0.034 ; online suppl. Tables 2,3$)$. In all, $57 \%$ of patients aged $\leq 40$ years had FC checked, compared to $12 \%$ aged $41-65$ and $0 \%$ aged $\geq 66$ years (online suppl. Tables 2, 3; guidelines advise to consider 2-week wait colonoscopies rather than the use of calprotectin in patients over 40 years).

\section{Clinical Outcomes and Disease Severity}

There was no significant difference in disease severity in the first year in either cohort (Table 4). The majority of patients requiring surgery in the first year had a diagnosis of CD ( $85.7 \%$ in $2013,75 \%$ in 2016$)$. Of those who required biologics, $\mathrm{CD}$ was the underlying subtype for $50 \%$ in 2013 (vs. $37.5 \%$ with UC) and 55\% in 2016 (vs. $45 \%$ with UC). Steroids were given at diagnosis to $57 \%$ with UC versus $34 \%$ with CD in 2013, and 60\% with UC versus $33 \%$ with CD in 2016 (remaining patients had IBDU).

When steroids were given, the time to treatment was 1.0 month in 2013 and 0.75 month in $2016(p=0.517)$. For biologics, time to first treatment was 1.5 months in 2013 and 0.61 month in $2016(p=0.287)$. For all surgery performed within 12 months of diagnosis, time to event was 2.0 months in 2013 and 1.5 months in 2016; however, all but 4 of these patients had surgery at diagnosis.

\section{Discussion}

Timely diagnosis of IBD and differentiation from IBS is crucial to good IBD management, yet this poses a diagnostic challenge for both primary care and IBD clinicians. FC is recommended by NICE to help distinguish between these 2 diagnoses $[9,10]$. The introduction of FC to primary care was intended to avoid unnecessary invasive tests in those patients unlikely to suffer from IBD and to help identify at-risk patients with potential IBD to expedite investigations and treatment. Numerous studies have exam- 
ined the diagnostic utility of FC in separating IBD from IBS in primary care [11, 13-15]. Our study is to our knowledge, however, the first attempt to investigate the association of primary care FC testing and time to diagnosis or treatment for those patients newly diagnosed with IBD.

When examining the newly diagnosed IBD patients in our centre looking after 770,000 inhabitants of Leeds, we found that neither time to diagnosis nor time to treatment improved significantly since the introduction of FC testing in primary care. Indeed, patients in the 2016 cohort with FC tests had similar time to diagnosis and treatment than those without. We found that time to diagnosis was not improved by the introduction of a primary care FC pathway for diagnosing IBD. However, many confounders make this difficult to interpret. It is important to recognize that $30 \%$ of patients present via the 2 -week wait pathway and $10 \%$ present as emergency admissions. These figures should be factored into service provision and planning. It is reassuring to observe the time to treatment amongst patients diagnosed via the 2 -week wait pathway decreased in 2016, as there is a real concern raised that once malignancy has been excluded, these patients are at risk of getting "lost" within the system and experiencing delays in treatment. Similarly, those diagnosed incidentally via the bowel screening programme had a statistically significant improvement in the time to treatment rate, confirming that the service has improved the pathway for these patients post-diagnosis.

There are considerable differences in time to diagnosis and treatment depending on referral routes. It is reassuring that the overall time to treatment is reduced in 2016 compared to 2013. However, it is particularly noticeable that referrals to surgical specialties cause the greatest amount of delay in time to diagnosis and time to treatment. This referral route should be discouraged by measuring FC levels where appropriate.

FC is likely to have contributed to the increased proportion of referrals directly to gastroenterology as intended by the introduction of the calprotectin referral pathway. This could be a positive outcome for patients as in some cases it may allow for earlier access to treatment and avoid unnecessary investigations. Direct referrals to gastroenterology also arguably improve patient experience as patients are not required to visit a number of different teams prior to commencing treatment, demonstrated by short time to treatment.

Overall, only $33.3 \%$ of new IBD diagnoses had FC checked. It is reassuring to observe that as intended by the local pathway, $88 \%$ of FC tests were conducted in the $\leq 40$ years age group. Whilst calprotectin testing is encouraged in patients with non-bloody diarrhoea under the age of 40 , we need to recognize that patients with bloody diarrhoea or in the appropriate age range for cancer referrals should not undergo calprotectin testing to avoid delays to colonoscopy [9]. There were a number of cases (age $\geq 60$ years or those with bloody diarrhoea) where FC was not indicated and may have delayed referral for a colonoscopy. In addition some patients with proctitis present with isolated rectal bleeding and may, therefore, be appropriately referred to surgical clinics first. Nevertheless, calprotectin testing needs to be more widely adopted in primary care to help identify high-risk patients. Further work is required to ensure appropriate use of this test, and it is hoped that initiatives such as the New Faecal Calprotectin Care Pathway will encourage this [10]. Despite this, it is recognized that identification of symptoms and patient concerns and expectations can prove a challenge for general practitioners. It is not always easy to encourage patients to provide a stool sample, and at times immediate referral is required - either due to severity of symptoms or the need to exclude red flag symptoms that might be suggestive of malignancy.

Of note, patients over 40 years who were referred via the suspected cancer pathways had a very short time to diagnosis. It would be desirable that suspected IBD would be given the same priority as suspected cancer in the diagnostic process.

A major strength of our study lies in the large cohort examined with comprehensive data collection and referral pathway characterization. Analysis of referrals to the IBD service at the Leeds Teaching Hospitals NHS Trust has demonstrated that patterns and prevalence of disease locally are in keeping with recognized statistics $[1,16,17]$. These data have highlighted a number of interesting learning points, and this study appears to be the first to investigate the association of FC with time to diagnosis and treatment of IBD in a pragmatic way.

There are a number of limitations to our study. Firstly, the patient data collected are dependent on the content and accuracy of documentation within referrals, endoscopy records, and clinic letters. Unfortunately, data on time to diagnosis were unavailable for patients who underwent endoscopic procedures at independent centres contracted to provide NHS care as the initial referral letters were not accessible for these patients. A further area of interest relates to duration of symptoms in the community prior to referral to secondary care and whether testing of FC has allowed more prompt referrals for further investigation. Data on duration of symptoms prior to referral were not readily available within letters, but investigating 
whether FC has reduced time from symptom onset to referral would be a future area of research interest, especially as diagnostic delays are associated with a more aggressive course of CD [18]. It is possible that a number of patients diagnosed with IBD were not identified by our search strategy, but this is likely to be a low number as referral to the IBD clinic is the locally agreed standard of care for all patients diagnosed within services commissioned by the NHS. It is possible that some patients diagnosed by private gastroenterologists or surgeons may not have been referred; however, this is frequently done for access to immunomodulator and biologic therapies. Furthermore, a number of findings showed only numerically rather than statistically significant differences. This likely relates to a lack of statistical power. As referral pathways differ between different locations in the UK, it would be very difficult to examine these associations in a multicentre study of very heterogeneous centres. Nevertheless, this study assessed for association in a real-world manner rather than causation. It is possible that other factors than the change in referral pathways may have contributed to our findings. However, the average waiting to first general gastroenterology appointments reduced during the study period, the number of IBD nurses and doctors participating in the IBD clinics increased, and clinic capacity was improved. Hence, it is unlikely that the lack of reduction in time to diagnosis and treatment relates to organizational changes within the gastroenterology department. Furthermore, time from referral to diagnosis was reasonable in 2013, and further improvements may have been difficult to achieve. Finally, local service set-ups differ between different geographical locations in the UK, never mind internationally; it is therefore unclear whether these results also apply to other regions. Despite this, we anticipate that most referral pathways will bear similarities to Leeds and that the broad findings are likely to be applicable throughout the UK.
In summary, this study highlights that FC testing can direct patients to the most appropriate service (direct referral to gastroenterology). In this cohort, we could not demonstrate improved outcomes for diagnosis and treatment of IBD when FC is tested. Further work, for instance through further training and education of primary care practitioners, is required to ensure patients with suspected IBD get referred to the most appropriate service in a timely manner.

\section{Statement of Ethics}

The study was conducted as a prospective clinical audit. Relevant clinical audit authorization was obtained. Due to the nature of this pragmatic service evaluation study, research ethics committee approval and informed consent were not required [19].

\section{Conflict of Interest Statement}

C.P.S. has received unrestricted research grants from Warner Chilcott, Janssen, and AbbVie; has provided consultancy to Warner Chilcott, Dr. Falk, AbbVie, Takeda, Fresenius Kabi, and Janssen; and had speaker arrangements with Warner Chilcott, Dr. Falk, AbbVie, MSD, Pfizer, and Takeda. All other authors declare no conflict of interest.

\section{Funding Sources}

No funding for this study was received.

\section{Author Contributions}

A.H. co-designed the study, collected the data, performed the analysis, and wrote the draft manuscript. C.P.S. designed the study, co-analysed the data, and critically reviewed the draft manuscript. P.J.H. planned the calprotectin pathway, critically reviewed the analysis, and critically reviewed the manuscript.

\section{References}

1 UK IBD standards. 2019

2 Lamb CA, Kennedy NA, Raine T, Hendy PA, Smith PJ, Limdi JK, et al. British Society of Gastroenterology consensus guidelines on the management of inflammatory bowel disease in adults. Gut. 2019;68(Suppl 3):s1106.

3 Vermeire S, Van Assche G, Rutgeerts P. Laboratory markers in IBD: useful, magic, or unnecessary toys? Gut. 2006;55(3):426-31.

4 Vavricka SR, Spigaglia SM, Rogler G, Pittet V, Michetti P, Felley C, et al. Systematic evaluation of risk factors for diagnostic delay in in- flammatory bowel disease. Inflamm Bowel Dis. 2012;18(3):496-505.

5 Cabrera-Abreu JC, Davies P, Matek Z, Murphy MS. Performance of blood tests in diagnosis of inflammatory bowel disease in a specialist clinic. Arch Dis Child. 2004;89(1):69-71.

6 Gallinger Z, Ungaro R, Colombel J-F, Sandler RS, Chen W. P030 delayed diagnosis of Crohn's disease is common and associated with an increased risk of disease complications. Inflamm Bowel Dis. 2019;25(Suppl 1):S14-5.

7 Cantoro L, Di Sabatino A, Papi C, Margagnoni G, Ardizzone S, Giuffrida P, et al. The time course of diagnostic delay in inflammatory bowel disease over the last sixty years: an Italian multicentre study. J Crohns Colitis. 2017; 11(8):975-80.

8 Mao R, Xiao YL, Gao X, Chen BL, He Y, Yang $\mathrm{L}$, et al. Fecal calprotectin in predicting relapse of inflammatory bowel diseases: a metaanalysis of prospective studies. Inflamm Bowel Dis. 2012;18(10):1894-9.

9 Faecal calprotectin diagnostic tests for inflammatory diseases of the bowel; Diagnostics guidance [DG11]. London: NICE; 2013. 
10 Turvill J, Turnock D, Holmes H, Jones A, McLaughlan E, Hilton V, et al. Evaluation of the clinical and cost-effectiveness of the York Faecal Calprotectin Care Pathway. Frontline Gastroenterol. 2018;9(4):285-94.

11 Kennedy NA, Clark A, Walkden A, Chang JC, Fascí-Spurio F, Muscat M, et al. Clinical utility and diagnostic accuracy of faecal calprotectin for IBD at first presentation to gastroenterology services in adults aged 16-50 years. J Crohns Colitis. 2015;9(1):41-9.

12 McFarlane M, Chambers S, Malik A, Lee B, Sung E, Nwokolo C, et al. Clinical outcomes at 12 months and risk of inflammatory bowel disease in patients with an intermediate raised fecal calprotectin: a 'real-world' view. BMJ Open. 2016;6(6):e011041.
13 Alibrahim B, Aljasser MI, Salh B. Fecal calprotectin use in inflammatory bowel disease and beyond: a mini-review. Can J Gastroenterol Hepatol. 2015;29(3):157-63.

14 Walsham NE, Sherwood RA. Fecal calprotectin in inflammatory bowel disease. Clin Exp Gastroenterol. 2016;9:21-9.

15 Jellema P, van Tulder MW, van der Horst HE, Florie J, Mulder CJ, van der Windt DA. Inflammatory bowel disease: a systematic review on the value of diagnostic testing in primary care. Colorectal Dis. 2011;13(3):239-54.
16 Mahid SS, Minor KS, Soto RE, Hornung CA, Galandiuk S. Smoking and inflammatory bowel disease: a meta-analysis. Mayo Clin Proc. 2006;81(11):1462-71.

17 Ye Y, Pang Z, Chen W, Ju S, Zhou C. The epidemiology and risk factors of inflammatory bowel disease. Int J Clin Exp Med. 2015;8(12): 22529-42.

18 Schoepfer AM, Dehlavi MA, Fournier N, Safroneeva E, Straumann A, Pittet V, et al. Diagnostic delay in Crohn's disease is associated with a complicated disease course and increased operation rate. Am J Gastroenterol. 2013;108(11):1744-54;

19 Defining research. In: Health Research Authority, editor. London: 2009. 